\title{
A Case Report on Prednisolone Induced Pedal Edema
}

\author{
P. Venkata Ramana ${ }^{1}$, S. Susmitha ${ }^{2 *}$,Danish Ahmad Khan², S K Eshma², S M D Yaseen ${ }^{2}$
}

${ }^{1}$ Assistant Professor, Department of Pharmacy Practice, P. Rami Reddy Memorial College of Pharmacy, Kadapa, Andhra Pradesh, INDIA.

${ }^{2}$ Pharm-D intern, Department of Pharmacy Practice, P. Rami Reddy Memorial College of Pharmacy, Kadapa, Andhra Pradesh INDIA.

\begin{abstract}
Prednisolone is a 1, 2-dehydrocortisolwhich is highly potent synthetic glucocorticosteroid which has minimal mineralocorticoid activity. Prednisolone is used as antinflammatory or immunosuppressive agent, which indicated in the treatment of various conditions, including congenital adrenal hyperplasia, psoriatic arthritis, systemic lupus erythematous, bullous dermatitis herpetiformis, seasonal or perennial allergic rhinitis. Majorly it causes Cushing Syndrome, peptic ulceration, edema, hypokalemia, muscle weakness, behavioral changes. A 55 year old female patient was consulted in the dermatology department with chief complaints of swelling and redness of blisters on mouth and fore head, difficulty while swallowing since 2 months, past medical history of patient includes, she was already taking treatment for this condition which includes Tab. Chlophenaramine malate $4 \mathrm{mg}$, Tab. Prednisolone 25 mg, Tab. Vitamin C 500 mg, Oint. Clobestalol $0.05 \%$ w/v, Glycerin lotion $5 \mathrm{ml}$. After 15 days, patient had complaints of swelling of both lower limbs after administration of Tab. Prednisolone, this is the reason for hospital admission. Better vigilance is necessary for implementation of safe and effective treatment for each individual patient. In-order to prevent serious adverse drug reactions of this drug, close monitoring during treatment course, creating awareness, recognition of the problem and careful management of all patients who receive this medication are essential, because use of corticosteroids causes toxic effects like abdominal tenderness, fluid retention, insomnia, muscle weakness etc. and if close monitoring is not provided during treatment course, it can cause permanent disability, morbidity, mortality.
\end{abstract}

Key words: Prednisolone, Pedal edema, Adverse drug reaction, Blisters.

\section{INTRODUCTION}

Prednisolone is a 1,2-dehydrocortisol which is highly potent synthetic glucocorticosteroid which has minimal mineralocorticoid activity. ${ }^{1}$ Prednisolone is used as anti-inflammatory or immunosuppressive agent, which indicated in the treatment of various conditions, including congenital adrenal hyperplasia, psoriatic arthritis, systemic lupus erythematous, bullous dermatitis herpetiformis, seasonal or perennial allergic rhinitis, allergic corneal marginal ulcers, symptomatic sarcoidosis, idiopathic thrombocytopenic purpura in adults, leukemias and lymphomas in adults, and ulcerative colitis. Glucocorticoids are adrenocortical steroids and cause profound and varied metabolic effects. In addition, they modify the body's immune responses to diverse stimuli. ${ }^{2}$ Majorly it causes Cushing Syndrome, peptic ulceration, edema, hypokalemia, muscle weakness, behavioral changes. ${ }^{3}$ This is the case report of 55 year old female patient who consulted dermatology department with stomatitis medicamentosa. Overall incidence rate of $5-7 \%$ in hospitalized patients. Almost any medicine can induce skin reactions, and certain drug classes, such as non-steroidal anti-inflammatory drugs (NSAIDs), antibiotics and antiepileptic have drug eruption rates approaching 1-5\%. Prednisolone is available in the form of oral and parental forms (IM). It readily absorbed by gastrointestinal tract, peak plasma concentration is 1-2 hours after administration. $90 \%$ shows high protein binding. Biological half-life of prednisolone is 2-3 hours, with $65 \%$ of excreted in the urine either in free form or glucoconjugate. ${ }^{4}$
DOI: 10.5530/ijopp.11.2.20

Address for correspondence: S. Susmitha Pharm. D intern, P. Rami Reddy Memorial College of Pharmacy, Kadapa, Andhra Pradesh, INDIA. Phone no: 8341503521 Email Id: sathyalasusmitha@ gmail.com

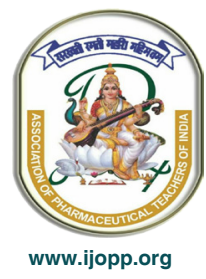




\section{CASE REPORT}

A 55 year old female patient was consulted in dermatology department with chief complaints of swelling and redness of blisters on mouth and fore-head, difficulty while swallowing since 2 months, past medical history of patient includes; she was already taking treatment for this condition which includes Tab. Chlophenaramine malate 4mg, Tab. Prednisolone 25mg, Tab. Vitamin C $500 \mathrm{mg}$, Oint. Clobestalol 0.05\% w/v, Glycerin lotion $5 \mathrm{ml}$. Again patient was consulted in dermatology department with complaints of swelling and itching at both lower limbs since 15 days. On general examination, patient was conscious and coherent. On physical examination, PR-82bpm, BP-130/80 mm/hg. On systemic examination, CVS- $\mathrm{S}_{1} \mathrm{~S}_{2}+$, RR-20 CPM, RS- clear, pedal edema at both lower limbs with $4 \mathrm{~mm}$ depth. On laboratory examination shows $\mathrm{Hb}: 11 \mathrm{gm} / \mathrm{dl}$, WBC: 9,500 cells/cumm, ESR: $28 \mathrm{~mm} / \mathrm{hr}$. The treatment was given as follows i.e.: Tab. Cetirizine 10mg, Tab. Vitamin C 500mg, and Glycerin lotion 5ml, Oint. Clobestalol $0.05 \% \mathrm{w} / \mathrm{v}$. Based on the above information here we have suspected it as possible ADR (pedal edema) shown in Figure1. Patient was referred to general medicine department to confirm the ADR. On analysis compared to all other drugs prescribed, prednisolone pharmacology and literature support the occurrence of edema. In order to conform the relationship between the effect and drug we have also done dechallenge test i.e. drug was withdrawn from the treatment regimen, and prescribed Tab. Furosemide 40mg, Tab. B. Complex 67mg.

\section{Causality assessment}

To evaluate the relationship between the drug and reaction, we have performed causality assessment by using scales like WHO causality assessment scale, naranjo's scale and karsch lasagna scale and analysis of observed ADR (Table 1) and (Table 2)

\section{DISCUSSION}

Edema may be defined as a clinically detectable increase in interstitial fluid volume. Edema develops when excess

\begin{tabular}{cccc}
\hline Table1: Causality assessment of suspected ADR. \\
\hline ADR SCALE & WHO-UMC & NARANJO'S & $\begin{array}{c}\text { KARSCH } \\
\text { and } \\
\text { LASAGNA }\end{array}$ \\
\hline ASSESSMENT & Probable & Probable & Probable \\
\hline
\end{tabular}

\begin{tabular}{cc}
\multicolumn{2}{c}{ Table 2: Analysis of observed ADR. } \\
\hline SEVERITY ASSESSMENT & Moderate level- 4 (a) \\
\hline PREVENTABILITY & Probably preventable \\
PREDICTABILITY & Type -A \\
\hline
\end{tabular}

Indian Journal of Pharmacy Practice, Vol 11, Issue 2, Apr-Jun, 2018

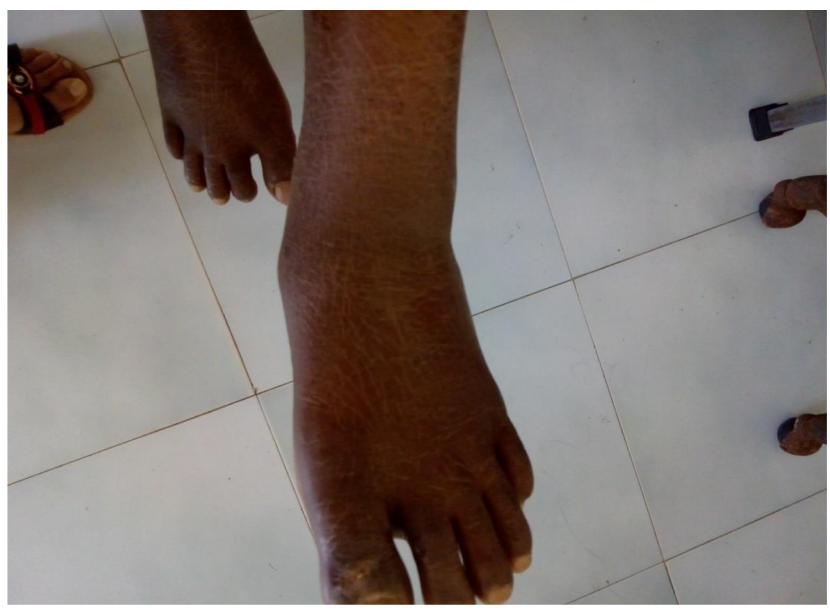

Figure1: Showing pedal edema.

sodium is retained either as a primary defect in renal sodium excretion, or as a response to a decrease in the effective circulating volume despite an already normal or expanded ECF volume. ${ }^{5}$ Edema can be explained by two ways localized edema and generalized edema, in localized edema it occurs due to venous or lymphatic obstruction allergic reactions and superior venacava obstruction are the causes of localized facial edema, generalized edema occurs due to soft tissue swelling of most of all regions of the body. ${ }^{6}$ Normally fluid will exist into interstitial from the arteriolar end of the microcirculation is nearly inflow of vascular end. Primarily sodium and water retention will takes place in renal finally there is an occurrence of edema. ${ }^{7}$ Edema is initially detected in feet or periorbial area in ambulatory patients and which is defined as pitting edema. ${ }^{8}$ Here Drug-induced edema can be divided into three types by the mechanism as follows, sodium overload, renal dysfunction and hyper permeability of blood vessel. In the category of sodium overload, edema is induced by much fluid replacement and antibiotics which contain large amount of sodium and sodium bicarbonate. As the category of renal dysfunction, NSAIDs, antihypertensive drugs, anticancer drugs and so on induce edema in patients with renal dysfunction. In the category of hyperpermeability of blood vessel, edema is induced by calcium antagonist, insulin. In our case Tab. Prednisolone was given to reduce inflammation at site of blisters occurs in mouth and forehead, but common adverse drug reactions like peptic ulceration, muscle weakness, insomnia, dry skin weight gain, fluid retention. ${ }^{9}$ In our case patient had complaints of swelling at both lower limbs this condition is called pedal edema. In our case patient had history of usage of Tab. Prednisolone and developed sever edema at both lower limbs. During treatment course as a clinical pharmacist we have identified adverse drug reactions as follows, the 
patient was under the medication with Tab. Prednisolone based upon the literature reviews and based on local examination and other investigations we have concluded that this condition is due to the drug Prednisolone and performed causality assessment, severity, preventability, predictability. After the identification we have immediately withdrawn the drug prednisolone and provided appropriate treatment.

\section{CONCLUSION}

Better vigilance is necessary for implementation of safe and effective treatment for each individual patient. In-order to prevent serious adverse drug reactions of this drug, close monitoring during treatment course, creating awareness, recognition of the problem and careful management of all patients who receive this medication are essential, because by the use of corticosteroids commonly causes toxic effects like abdominal tenderness, fluid retention, insomnia, muscle weakness etc. if not providing close monitoring during treatment course, which can cause permanent disability, morbidity, mortality.

\section{CONFLICT OF INTEREST}

The authors declare no conflict of interest.

\section{ABBREVIATIONS}

ADR: Adverse Drug Reaction; ADE: Adverse Drug Event; WHO: World Health Organization; NSAIDS:
Non Steriodal Anti Inflammatary Drugs; Ecf: Extracellular Fluid.

\section{ACKNOWLEDGEMENT}

I would like to express my special thanks of gratitude to my teacher Dr. P. venkata ramana.

\section{SUMMARY}

Glucocorticoids(prednisolone in dose of above $5 \mathrm{mg}$ ) have weak mineralocorticoid action, cause sodium and water retention, promotes potassium excretion.thus prolonged use of these drugs may cause pedal oedema.

\section{REFERENCES}

1. Tripati KD.Essentials of medical pharmacology, Jaypee the health science publishers.2013:(7):295-6.

2. Sharma $\mathrm{HL}$, Sharma KK. Principles of pharmacology, Paras medicPublishers.2013:(2):630.

3. Tara Shanbhag.Basic pharmacology and pre manual under graduates, Elsieverpublishers. 2015:(4):334-7.

4. Rang and Dales, Text book of Pharmacology, Elsevier publishers.2017;(7):409.

5. Joseph T. DiproA text book of Pharmacotherapy; a pathophysiologic approach.2012:(9):856.

6. Harrisons. Principles of internal medicine, MC Graw hill. 2013:(1):150.

7. Robbins, Basic pathology, Elsevier publisher's. 2005:(7):80.

8. Roger walker, Cate whittlesea, Clinical pharmacy and therapeutics,Elsevier publishers. 2015:(5):286.

9. Brain R. Walker, Davidson's Principles and practice of medicine, ElsevierPublishers. 2014:(22):478 